\title{
IUFOST2006/1191 \\ French National Research Agency (ANR) : Research programme and actions in Food and Human Nutrition
}

\author{
M. Marin \\ Agence Nationale de la Recherche, 22 Rue Monge, 75005 PARIS, France \\ michele.marin@agencerecherche.fr
}

French National Research Agency (ANR) : Priorities in Food and Human Nutrition, programmes and instruments, coordination with European research areas (ERA-Net, ...)

ANR has been created in 2004 to stimulate French research, bring more flexibility, reactivity and, as a consequence, competitiveness while keeping a good balance between fundamental research and applied research. ANR complements the recurrent funding of the research organisations with a funding system based on objectives and projects. Two calls have been launched in the field of Food and Human Nutrition (2005 and 2006) based on 5 research priorities, funding both academic projects and public-private partnership projects. Thematic priorities and figures illustrating the Food an Human Nutrition calls would be presented. The aim of ANR would also be to develop synergy and complementarities of national /EC programmes and instruments. The objectives for 2010 are to devote $20 \%$ of the budget in international calls for proposals through Bilateral and multilateral cooperation and ERA-NET. Future actions in Food and Human Nutrition would be discussed. 